\title{
Thermal behaviour of metal films - a hyperbolic two-step model
}

\author{
M. A. Al-Nimr, 0. M. Haddad, V. S. Arpaci
}

Abstract A perturbation technique is proposed for solution of the generalized equations governing the thermal behaviour of thin metal films described by a hyperbolic two-step model. The generalized equations of this model contains diffusion terms in both the electron and lattice energy equations and assumes that incident laser radiation is absorbed by both the electron gas and solid lattice to account for the thermal behaviour of semiconducting and impure metals. A perturbation technique is utilized to eliminate the coupling between the electron and phonon energy equations when the normalized temperature difference between electrons and phonons is a small quantity, which is true in materials exhibit high coupling factors.

\section{List of symbols}

$\begin{array}{ll}C & \text { heat capacity } \mathrm{Jm}^{-3} \mathrm{~K}^{-1} \\ C_{R} & \text { heat capacity ratio, } C_{\mathrm{e}} / C_{\mathrm{l}} \\ G & \text { electron-phonon coupling factor, } \mathrm{Wm}^{-3} \mathrm{~K}^{-1} \\ G & \text { Green's function } \\ \mathrm{h} & \text { Planck constant, } \mathrm{J} \\ k_{\mathrm{B}} & \text { Boltzmann constant, } \mathrm{J} \mathrm{K}^{-1} \\ K & \text { thermal conductivity } \mathrm{Wm}^{-1} \mathrm{~K}^{-1} \\ K_{\mathrm{R}} & \text { thermal conductivity ratio, } K_{\mathrm{e}} / K_{\mathrm{l}} \\ L & \text { film thickness, m } \\ m^{\mathrm{e}} & \text { effective mass of electrons, kg } \\ n_{\mathrm{e}} & \text { electron number density per unit volume } 1 / \mathrm{m}^{3} \\ n_{\mathrm{a}} & \text { atomic density per unit volume, } 1 / \mathrm{m}^{3} \\ \mathrm{~N} & \text { number of atoms in the solid } \\ P_{\mathrm{e}} & \text { dimensionless heat source in the electron gas, } \\ & S_{\mathrm{e}} L^{2} / T_{\mathrm{i}} K_{\mathrm{e}} \\ P_{1} & \text { dimensionless heat source in the lattice, } S_{\mathrm{l}} L^{2} C_{R} / T_{\mathrm{i}} K_{\mathrm{e}} \\ q & \text { conduction heat flux, Wm } \\ Q & \text { dimensionless conduction heat flux, } q L / T_{\mathrm{i}} K_{\mathrm{e}} \\ S_{\mathrm{e}} & \text { volumetric heat source in the electron gas, Wm } \mathrm{Wm}^{-3} \\ S_{\mathrm{l}} & \text { volumetric heat source in the lattice, } \mathrm{Wm} \mathrm{m}^{-3} \\ t & \text { time, s }\end{array}$

Received on 6 January 1999

M. A. Al-Nimr, O. M. Haddad

Mechanical Engineering Department

Jordan University of Science and Technology

P.O. Box 3030, Irbid 22110, Jordan

V. S. Arpaci

Mechanical Engineering Department

University of Michigan, Ann Arbor, USA

Correspondence to: M. A. Al-Nimr temperature, $\mathrm{K}$

$T_{\mathrm{D}} \quad$ Debye temperature, $\mathrm{K}$

$T_{\mathrm{i}} \quad$ initial temperature of both lattice and electron gas, $\mathrm{K}$ speed of sound, $\mathrm{m} \mathrm{s}^{-1}$

spatial coordinate, $\mathrm{m}$

dimensionless spatial coordinate, $x / L$

\section{Greek symbols}

$\delta \quad$ Dirac's delta function

$\Delta \quad$ difference function

$\epsilon \quad$ dimensionless small parameter

dimensionless temperature, $\left(T-T_{\mathrm{i}}\right) / T_{\mathrm{i}}$

dimensionless time, $t K_{\mathrm{e}} /\left(L^{2} C_{\mathrm{e}}\right)$

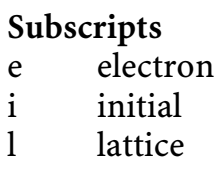

1

\section{Introduction}

In applications involving high-rate heating induced by a short-pulse laser, the typical response time is an order of picoseconds (Qiu and Tien, 1992, 1993) which is comparable to the phonon-electron thermal relaxation time. In such cases, thermal equilibrium between phonons and electrons cannot be assumed and heat transfer in the electron gas and the metal lattice needs to be considered separately. Models describing the non-equilibrium thermal behavior in such cases are called the microscopic two-step models. In the literature, there are two microscopic twostep models. The first one is the parabolic two-step model which is pioneered by Anisimov et al. (1974) and advanced later by Fujimoto et al. (1984). The second one is the hyperbolic two-step model introduced by Qiu and Tien (1993) based on the macroscopic averages of the electric and heat currents carried by electrons in the momentum space. The parabolic two-step model involves two coupled energy equations governing the heat transfer in the electron-gas and the metal-lattice. Solutions for this model can be found in the literature for different operating and boundary conditions and using different solutions methodologies (Al-Nimr, 1997; Al-Nimr and Masoud, 1997; Tzou et al., 1994; Tzou, 1995a). On the other hand, the hyperbolic two-step model, in its simplest form, involves three coupled equations. This model does not account for the conduction of heat by phonons and for the absorption of the incident laser energy by the electron gas (Tzou, 
1995b). When these effects are included, the model leads to four coupled equations which are difficult to solve.

The electron-phonon coupling varies over a wide range. Some metals have low and some others have high coupling factors. For example, the coupling factor of gold is about $2.6 \times 10^{16} \mathrm{Wm}^{-1} \mathrm{~K}^{-1}$ and that of Vanadium $(\mathrm{V})$ is about $648 \times 10^{16} \mathrm{Wm}^{-3} \mathrm{~K}^{-1}$. As the value of the coupling factor increases, the thermalization time (the time required for the electron and lattice to reach equilibrium state) decreases (Tzou, 1995a, b). Before thermalization, the normalized temperature difference between the electron gas and the lattice may be small but not negligible. This small difference is observed especially in materials having large coupling factors and when the laser duration time is long enough to enable the electron gas to give part of its energy to the solid lattice. When the temperature difference between the electron gas and the lattice is small enough, the difference may be normalized as a perturbed quantity. Then, a perturbation technique may be used to eliminate the coupling between the energy equations. The elimination of this coupling produces four uncoupled equations which have the same order as the original coupled equations.

The objective of this work is to reformulate the generalized governing equations of the hyperbolic two-step model by a perturbation technique. These equations include diffusion terms in both electron gas and solid lattice describing the thermal behavior of semiconductors and metals with impurities. Also, the generalized equations assume that incident laser heating is absorbed by both electron gas and solid lattice. As an illustrative example, a simple problem is solved analytically by the proposed perturbation technique. Also, a criterion which determine the parameters affecting the thermal equilibrium is derived.

\section{2}

\section{Analysis}

Qiu and Tien (1993) derived the hyperbolic two-step radiation heating model based on the macroscopic averages of the electric and heat currents carried by electrons in the momentum space. In the absence of electric current during laser heating, they arrived at three coupled equations describing the one-dimensional energy exchange between phonons and electrons. These equations, in their dimensionless form, are written as:

$$
\begin{aligned}
& \frac{\partial \theta_{\mathrm{e}}}{\partial \tau}=-\frac{\partial Q_{\mathrm{e}}}{\partial X}-H_{1}\left(\theta_{\mathrm{e}}-\theta_{\mathrm{l}}\right)+P_{\mathrm{e}} \\
& \frac{\partial \theta_{\mathrm{l}}}{\partial \tau}=C_{R} H_{1}\left(\theta_{\mathrm{e}}-\theta_{\mathrm{l}}\right) \\
& \frac{\partial Q_{\mathrm{e}}}{\partial \tau}+H_{2} \frac{\partial \theta_{\mathrm{e}}}{\partial X}+H_{2} Q_{\mathrm{e}}=0
\end{aligned}
$$

These equations assume that electronic contribution to thermal conductivity dominates the phonon contribution and that the incident radiation is totally absorbed by the electron gas. This is true especially in pure metals in which the electronic contribution is usually more than $90 \%$ of the total conductivity (Blakemore, 1974). In the present work, Eqs. (1)-(3) are generalized by assuming that the conduction of heat by phonons is significant and that the incident laser energy is absorbed by both electron gas and solid lattice. Assumption that conduction of heat may be carried by phonons, as well as by electrons, is justified for metals containing significant impurities or for semiconductors in which the energy is diffused by both solid lattice and electron gas. It is also known that energy deposits into materials in different ways, depending on the nature of heating methods and the structure of materials. For example, energy can be deposited simultaneously on both electrons and phonons, through contact heating at surfaces, or selectively on a particular group of carriers by radiative heating. Radiation excites free/bound electrons in metals, but excites electrons or phonons in semiconductors. So it is necessary for each one of the energy equations of the hyperbolic two-step models to contain a source term to account for that part of incident radiation absorbed by electrons and phonons.

On the basis of the foregoing discussion, the governing equations (1)-(3) are:

$$
\begin{aligned}
& \frac{\partial \theta_{\mathrm{e}}}{\partial \tau}=-\frac{\partial Q_{\mathrm{e}}}{\partial X}-H_{1}\left(\theta_{\mathrm{e}}-\theta_{\mathrm{l}}\right)+P_{\mathrm{e}} \\
& \frac{\partial \theta_{\mathrm{l}}}{\partial \tau}=-C_{R} \frac{\partial Q_{\mathrm{l}}}{\partial X}+C_{R} H_{1}\left(\theta_{\mathrm{e}}-\theta_{\mathrm{l}}\right)+P_{1} \\
& \frac{\partial Q_{\mathrm{e}}}{\partial \tau}+H_{2} \frac{\partial \theta_{\mathrm{e}}}{\partial X}+H_{2} Q_{\mathrm{e}}=0 \\
& \frac{\partial Q_{\mathrm{l}}}{\partial \tau}+H_{3} \frac{\partial \theta_{\mathrm{l}}}{\partial X}+H_{2} Q_{\mathrm{l}}=0
\end{aligned}
$$

where

$$
\begin{aligned}
& H_{1}=\frac{L^{2} G}{K_{\mathrm{e}}}, \quad H_{2}=\frac{L^{2} C_{\mathrm{e}}}{K_{\mathrm{e}} \tau_{F}}, \quad H_{3}=\frac{H_{2}}{K_{R}}, \quad C_{R}=\frac{C_{\mathrm{e}}}{C_{\mathrm{l}}}, \\
& K_{R}=\frac{K_{\mathrm{e}}}{K_{\mathrm{l}}}, \quad P_{\mathrm{e}}=\frac{S_{\mathrm{e}} L^{2}}{T_{\mathrm{i}} K_{\mathrm{e}}}, \quad P_{1}=\frac{S_{\mathrm{l}} L^{2} C_{R}}{T_{\mathrm{i}} K_{\mathrm{e}}},
\end{aligned}
$$

The initial and boundary conditions to be satisfied are: For $\tau=0: \theta_{\mathrm{e}}=\theta_{\mathrm{l}}=Q_{\mathrm{e}}=Q_{\mathrm{l}}=0$.

$$
\begin{aligned}
& X=0: \frac{\partial \theta_{\mathrm{e}}}{\partial X}=\frac{\partial \theta_{1}}{\partial X}=0 \\
& X=1: \frac{\partial \theta_{\mathrm{e}}}{\partial X}=\frac{\partial \theta_{1}}{\partial X}=0
\end{aligned}
$$

Equations (4)-(7) are four coupled equations. Elimination of the coupling between these equations yields four uncoupled equations which contain mixed derivatives. The higher order and mixed derivatives resulting from this elimination raise the difficulties encountered in solution. However, in many cases, the coupling between the energy equations (4)-(7) may be eliminated without raising the order of the resulting equations and without creating mixed derivatives. These cases involve situations in which the incident radiation interacts with materials having very large coupling factor or cases in which the laser pulse duration is not too short. In these cases, the difference between the electron and lattice temperature may be written as a perturbation,

$\theta_{\mathrm{e}}(\tau, \xi)=\theta_{\mathrm{l}}(\tau, \xi)+\epsilon \Delta(\tau, \xi)$ 
where $\Delta(\tau, \xi)$ is a function of space and time, and $\epsilon=1 / H_{1}$ is a dimensionless small parameter. Consider, for an example, the interaction of laser beam with a very thin lead film of $1 \times 10^{-6} \mathrm{~m}$ thickness. For lead, the thermal conductivity and coupling factor have values of $35 \mathrm{Wm}^{-1} \mathrm{~K}^{-1}$ and $12.4 \times 10^{16} \mathrm{Wm}^{-3} \mathrm{~K}^{-1}$, respectively (Qiu and Tien, 1992; Tzou, 1995 a, b). Under these conditions, $H_{1}=3500$ and a result, $\epsilon\left(=1 / H_{1}\right)$ may be considered as a very small perturbed quantity. Examples of other metals having very large coupling factor (i.e., having very small perturbed parameter $\epsilon$ ) are Vanadium, Niobium,

Titanium, etc.

Elimination of $\theta_{\mathrm{e}}-\theta_{1}$ between Eqs. (4) and (5), using Eq. (8), and droping terms of order $\epsilon$ and higher, yields:

$\frac{\partial \theta_{\mathrm{e}}}{\partial \tau}=-\Gamma_{1}\left[\frac{\partial Q_{\mathrm{e}}}{\partial X}+\frac{\partial Q_{1}}{\partial X}\right]+\Gamma_{2} P_{\mathrm{e}}+\Gamma_{3} P_{1}+\mathrm{O}(\epsilon)$

where

$\Gamma_{1}=\frac{C_{R}}{1+C_{R}}, \quad \Gamma_{2}=\Gamma_{1}, \quad \Gamma_{3}=\frac{\Gamma_{1}}{C_{R}}$

Now, differentiating both Eqs. (6) and (7) with respect to $X$, and combining the results with regard to Eq. (8), yield,

$$
\begin{aligned}
\frac{\partial^{2}}{\partial \tau \partial X}\left[Q_{e}+Q_{1}\right] & +\left(H_{2}+H_{3}\right) \frac{\partial^{2} \theta_{\mathrm{e}}}{\partial X^{2}} \\
& +H_{2} \frac{\partial}{\partial X}\left[Q_{\mathrm{e}}+Q_{1}\right]=\mathrm{O}(\epsilon)
\end{aligned}
$$

Elimination $\left[Q_{\mathrm{e}}+Q_{1}\right] \mathrm{\partial} / \partial X$ between Eqs. (9) and (10) gives:

$$
\begin{aligned}
\frac{\partial^{2}}{\partial \tau \partial X}\left[Q_{\mathrm{e}}+Q_{1}\right] & +\left(H_{2}+H_{3}\right) \frac{\partial^{2} \theta_{\mathrm{e}}}{\partial X^{2}}-\frac{H_{2}}{\Gamma_{1}} \frac{\partial \theta_{\mathrm{e}}}{\partial \tau}+\frac{H_{2} \Gamma_{2}}{\Gamma_{1}} P_{\mathrm{e}} \\
& +\frac{H_{2} \Gamma_{3}}{\Gamma_{1}} P_{1}=\mathrm{O}(\epsilon)
\end{aligned}
$$

Differentiating Eq. (9) with respect to $\tau$ yields:

$\frac{\partial^{2} \theta_{\mathrm{e}}}{\partial \tau^{2}}=-\Gamma_{1} \frac{\partial^{2}}{\partial \tau \partial X}\left[Q_{\mathrm{e}}+Q_{1}\right]+\Gamma_{2} \frac{\partial P_{\mathrm{e}}}{\partial \tau}+\Gamma_{3} \frac{\partial P_{1}}{\partial \tau}$

Eliminating $\partial^{2}\left[Q_{e}+Q_{1}\right] / \partial \tau \partial X$ between Eqs. (11) and (12) gives

$\frac{\partial^{2} \theta_{\mathrm{e}}}{\partial \tau^{2}}+H_{2} \frac{\partial \theta_{\mathrm{e}}}{\partial \tau}-\Gamma_{4} \frac{\partial^{2} \theta_{\mathrm{e}}}{\partial X^{2}}-H_{2} S-\frac{\partial S}{\partial \tau}=\mathrm{O}(\epsilon)$

where

$\Gamma_{4}=\Gamma_{1}\left(H_{2}+H_{3}\right), \quad S=\Gamma_{2} P_{\mathrm{e}}+\Gamma_{3} P_{1}$

After solving for $\theta_{\mathrm{e}}$ from Eq. (13), $Q_{\mathrm{e}}$ may be obtained from (6) as:

$Q_{\mathrm{e}}(\tau, X)=-\mathrm{e}^{-H_{2} \tau} \int_{0}^{\tau} H_{2} \frac{\partial \theta_{\mathrm{e}}}{\partial X} \mathrm{e}^{H_{2} \tau} \mathrm{d} \tau$

In Eq. (14), it is assumed that there is no conduction of heat by the electron gas at $\tau=0$. Also, substituting for $\theta_{\mathrm{e}}-\theta_{\mathrm{l}}$ from (8) into (4) yields

$\Delta(\tau, X)=-\frac{\partial \theta_{\mathrm{e}}}{\partial \tau}-\frac{\partial Q_{\mathrm{e}}}{\partial X}+P_{\mathrm{e}}$

and the $\theta_{1}$ is obtained from (8) as:
$\theta_{\mathrm{l}}=\theta_{\mathrm{e}}-\frac{\Delta}{H_{1}}$

Now, $Q_{1}$ is obtained from Eq. (7) as

$Q_{1}(\tau, X)=-H_{3} \mathrm{e}^{-k_{R} H_{3} \tau} \int_{0}^{\tau} \frac{\partial \theta_{1}}{\partial X} \mathrm{e}^{k_{R} H_{3} \tau} \mathrm{d} \tau$

Also, it is assumed that there is no conduction of heat by the solid lattice at $\tau=0$.

The analytical solution to Eq. (13) is expressed in terms of Green's function (see, for example, Ozisik, 1993)

$$
\begin{aligned}
\theta_{\mathrm{e}}(\tau, X)= & \int_{X^{\prime}=0}^{1} \int_{\tau^{*}=0}^{\tau} G\left(X, \tau \mid X^{\prime}, \tau^{*}\right) \\
& \times\left[H_{2} S\left(X^{\prime}, \tau^{*}\right)+\frac{\partial S}{\partial \tau^{*}}\left(X^{\prime}, \tau^{*}\right)\right] \mathrm{d} \tau^{*} \mathrm{~d} X^{\prime}
\end{aligned}
$$

Here $G\left(X, \tau \mid X^{\prime}, \tau^{*}\right)$ is the appropriate Green's function for the solution of governing equations (13)and its initial and boundary conditions with the source term replaced by the unit impulse function $\delta\left(\tau-\tau^{*}\right) \delta\left(X-X^{\prime}\right)$. In terms of the Green function, the electron temperature given by Eq. (13) satisfies

$\frac{\partial^{2} G}{\partial \tau^{2}}+H_{2} \frac{\partial G}{\partial \tau}-\Gamma_{4} \frac{\partial^{2} G}{\partial X^{2}}=\delta\left(\tau-\tau^{*}\right) \delta\left(X-X^{\prime}\right)$

Subject to the following initial and boundary conditions:

$\frac{\partial G}{\partial X}=0, \quad X=0$
$\frac{\partial G}{\partial X}=0, \quad X=1$
$G=0, \quad \tau<\tau^{*}$
$\frac{\partial G}{\partial \tau}=0, \quad \tau<\tau^{*}$

The initial conditions are based on the causality principle, which states that there can be no effect experienced at times prior to the cause. The finite integral transforms are used to solve system (19) and (20). Consider the integral transform and inversion pair defined as (see, for example, Ozisik, 1993):

$\bar{G}_{m}(\tau)=\int_{X^{\prime}=0}^{1} G\left(X, \tau \mid X^{\prime}, \tau^{*}\right) \cos \left(\lambda_{m} X\right) \mathrm{d} X$

$G\left(X, \tau \mid X^{\prime}, \tau^{*}\right)=\sum_{m=0}^{\infty} \frac{\bar{G}_{m}(\tau)}{N\left(\lambda_{m}\right)} \cos \left(\lambda_{m} X\right)$

where $N\left(\lambda_{m}\right)$ is the normalization integral given by

$N\left(\lambda_{m}\right)=1$ for $m=0$ and

$N\left(\lambda_{m}\right)=0.5$ for $m=1,2,3, \ldots$

and $\lambda_{m}$ 's are the eigenvalues by

$\lambda_{m}=m \pi$

The transform of Eq. (19) satisfies

$$
\begin{aligned}
& \frac{\mathrm{d}^{2} \bar{G}_{m}(\tau)}{\mathrm{d} \tau^{2}}+H_{2} \frac{\mathrm{d} \bar{G}_{m}(\tau)}{\mathrm{d} \tau}+\lambda_{m}^{2} \Gamma_{4} \bar{G}_{m}(\tau) \\
& \quad=\cos \left(\lambda_{m} X^{\prime}\right) \delta\left(\tau-\tau^{*}\right)
\end{aligned}
$$


subject to

$$
\begin{aligned}
& \bar{G}_{m}(\tau)=0, \quad \tau<\tau^{*} \\
& \frac{\mathrm{d} \bar{G}_{m}(\tau)}{\mathrm{d} \tau}=0, \quad \tau<\tau^{*}
\end{aligned}
$$

By a somewhat lengthy but straightforward manipulations, the solution to the system of equations (25) and (26) leads to

$\bar{G}_{m}(\tau)=\frac{\cos \left(\lambda_{m} X^{\prime}\right)}{\beta_{m}} \mathrm{e}^{-\frac{H_{2}}{2}\left(\tau-\tau^{*}\right)} \sin \left(\beta_{m}\left(\tau-\tau^{*}\right)\right), \quad \tau>\tau^{*}$

where $\beta_{m}=\sqrt{\Gamma_{4} \lambda_{m}^{2}-\frac{H_{2}^{2}}{4}}$. The inversion formula give by Eq. (22) readily gives the Green function as

$$
\begin{aligned}
G\left(X, \tau \mid X^{\prime}, \tau^{*}\right)= & \frac{2}{H_{2}} \mathrm{e}^{-\frac{H_{2}}{2}\left(\tau-\tau^{*}\right)} \sinh \left[\frac{H_{2}}{2}\left(\tau-\tau^{*}\right)\right] \\
& +\sum_{m=1}^{\infty} \frac{2 \cos \left(\lambda_{m} X^{\prime}\right)}{\beta_{m}} \mathrm{e}^{-\frac{H_{2}}{2}\left(\tau-\tau^{*}\right)} \\
& \times \sin \left(\beta_{m}\left(\tau-\tau^{*}\right)\right) \cos \left(\lambda_{m} X\right), \quad \tau>\tau^{*}
\end{aligned}
$$

In terms of this solution, Eq. (18) yields the electron temperature,

$$
\begin{aligned}
\theta_{\mathrm{e}}(X, \tau)=\int_{X^{\prime}=0}^{1} & \int_{\tau^{*}=0}^{\tau} \frac{2}{H_{2}} \mathrm{e}^{-\frac{H_{2}}{2}\left(\tau-\tau^{*}\right)} \sinh \left[\frac{H_{2}}{2}\left(\tau-\tau^{*}\right)\right] \\
& \times\left[H_{2} S\left(X^{\prime}, \tau^{*}\right)+\frac{\partial S}{\partial \tau^{*}}\left(X^{\prime}, \tau^{*}\right)\right] \mathrm{d} \tau^{*} \mathrm{~d} X^{\prime} \\
+\sum_{m=1}^{\infty} & \int_{X^{\prime}=0}^{1} \int_{\tau^{*}=0}^{\tau} \frac{2 \cos \left(\lambda_{m} X^{\prime}\right)}{\beta_{m}} \mathrm{e}^{-\frac{H_{2}}{2}\left(\tau-\tau^{*}\right)} \\
& \times \sin \left(\beta_{m}\left(\tau-\tau^{*}\right)\right) \cos \left(\lambda_{m} X\right) \\
& \times\left[H_{2} S\left(X^{\prime}, \tau^{*}\right)+\frac{\partial S}{\partial \tau^{*}}\left(X^{\prime}, \tau^{*}\right)\right] \mathrm{d} \tau^{*} \mathrm{~d} X^{\prime}
\end{aligned}
$$

The first term on the RHS of Eq. (29) represents the steady-state part of the solution remaining after the transients have died out. Any energy released within any insulated region will merely distribute evenly over the entire region after sufficient time. To proceed further, the nature of the heating source needs to be specified. Now, assume an impulse energy source, which is totally absorbed by the electron gas, released at time $\tau=0$ and at location $X=0$, and given by

$S(\tau, X)=S_{0} \delta(\tau) \delta(X)$,

where $S_{0}=\Gamma_{2}, \Gamma_{3}=0$ and $\delta$ is the Dirac's delta function. Such an energy source could model, for example, the application of strong laser pulses at the boundary of an absorbing medium which is encountered in the annealing of semiconductors (see, for example, Appleton and Cellar, 1982).
Now, with the notation that

$\int_{X^{\prime}=0}^{1} f\left(\tau, \tau^{*}, X, X^{\prime}\right) \delta\left(X^{\prime}-X_{0}\right) \mathrm{d} X^{\prime}=f\left(\tau, \tau^{*}, X, X_{0}\right)$

$$
\begin{aligned}
& \int_{\tau^{*}=0}^{\tau} f\left(\tau, \tau^{*}, X, X^{\prime}\right) \delta\left(\tau^{*}-\tau_{0}\right) \mathrm{d} \tau^{*}=f\left(\tau, \tau_{0}, X, X^{\prime}\right) \\
& \int_{\tau^{*}=0}^{\tau} f\left(\tau, \tau^{*}, X, X^{\prime}\right) \frac{\partial \delta}{\partial \tau^{*}}\left(\tau^{*}-\tau_{0}\right) \mathrm{d} \tau^{*} \\
& =\left[f\left(\tau, \tau^{*}, X, X^{\prime}\right) \delta\left(\tau^{*}-\tau_{0}\right)\right]_{\tau^{*}=0}^{\tau} \\
& \quad-\int_{\tau^{*}=0}^{\tau} \frac{\partial f}{\partial \tau^{*}}\left(\tau, \tau^{*}, X, X^{\prime}\right) \delta\left(\tau^{*}-\tau_{0}\right) \mathrm{d} \tau^{*}
\end{aligned}
$$

and for $\tau_{0}=0$, Eq. (33) is reduced to:

$$
\begin{aligned}
\int_{\tau^{*}=0}^{\tau} & f\left(\tau, \tau^{*}, X, X^{\prime}\right) \frac{\partial \delta}{\partial \tau^{*}}\left(\tau^{*}-\tau_{0}\right) \mathrm{d} \tau^{*} \\
= & -\int_{\tau^{*}=0}^{\tau} \frac{\partial f}{\partial \tau^{*}}\left(\tau, \tau^{*}, X, X^{\prime}\right) \delta\left(\tau^{*}-\tau_{0}\right) \mathrm{d} \tau^{*} \\
= & -\left.\frac{\partial f}{\partial \tau^{*}}\left(\tau, 0, X, X^{\prime}\right)\right|_{\tau^{*}=0}
\end{aligned}
$$

As a result, the temperature distribution is directly available by inserting Eq. (30) into the general solution (29), after performing indicated operations as in Eqs. (31)-(34),

$$
\begin{aligned}
\theta_{\mathrm{e}}(X, \tau)=S_{0}\{1 & +\sum_{m=1}^{\infty} \mathrm{e}^{-\frac{H_{2}}{2} \tau} \cos \left(\lambda_{m} X\right) \\
\times & {\left.\left[\frac{H_{2}}{\beta_{m}} \sin \left(\beta_{m} \tau\right)+2 \cos \left(\beta_{m} \tau\right)\right]\right\} }
\end{aligned}
$$

Also, $Q_{e}$ is found after inserting Eq. (35) into Eq. (14),

$Q_{\mathrm{e}}(X, \tau)=2 H_{2} S_{0} \sum_{m=1}^{\infty} \frac{\lambda_{m}}{\beta_{m}} \mathrm{e}^{-\frac{H_{2}}{2} \tau} \sin \left(\lambda_{m} X\right) \sin \left(\beta_{m} \tau\right)$

and $\Delta$ is found in terms of Eqs. (15), (35) and (36) as:

$\Delta(X, \tau)=\sum_{m=1}^{\infty} S_{0} \gamma_{m} \mathrm{e}^{-\frac{H_{2}}{2} \tau} \cos \left(\lambda_{m} X\right) \sin \left(\beta_{m} \tau\right)+P_{\mathrm{e}}(\tau, X)$

where $\gamma_{m}=2 \beta_{m}+\left(H_{2}^{2} / 2 \beta_{m}\right)-\left(2 H_{2} \lambda_{m}^{2} / \beta_{m}\right)$. Also, $\theta_{\mathrm{l}}$ is found from Eqs. (16), (35) and (37),

$$
\begin{aligned}
\theta_{\mathrm{l}}(X, \tau)= & S_{0}-\frac{P_{\mathrm{e}}}{H_{1}}+\sum_{m=1}^{\infty} S_{0} \mathrm{e}^{-\frac{H_{2}}{2} \tau} \cos \left(\lambda_{m} X\right) \\
& \times\left[\left(\frac{H_{2}}{\beta_{m}}-\frac{\gamma_{m}}{H_{1}}\right) \sin \left(\beta_{m} \tau\right)+2 \cos \left(\beta_{m} \tau\right)\right]
\end{aligned}
$$

A sample of the results will be presented for a thin metal film exposed to a laser pulse as in Eq. (30). The metal film is insulated from both sides and its thermal behavior is described by Eqs. (4)-(7) and their initial and boundary conditions. The schematic diagram of the problem is shown in Fig. 1. The results are obtained using the following values for different parameters: 


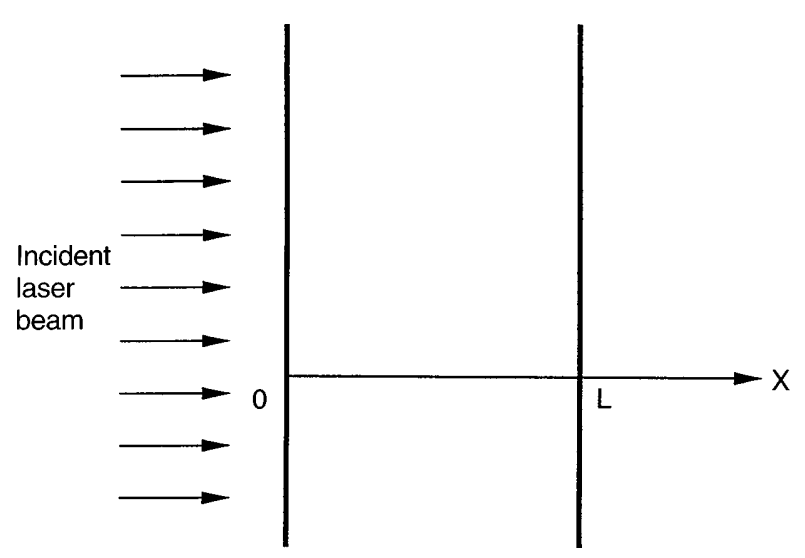

Fig. 1. Schematic diagram

$$
\begin{aligned}
& L=5 \times 10^{-6} \mathrm{~m}, \quad G=12.4 \times 10^{16} \mathrm{Wm}^{-3} \mathrm{~K}^{-1}, \\
& K_{\mathrm{e}}=35 \mathrm{Wm}^{-1} \mathrm{~K}^{-1}, \quad K_{\mathrm{l}}=0 \mathrm{Wm}^{-1} \mathrm{~K}^{-1}, \quad T_{\mathrm{i}}=300 \mathrm{~K}, \\
& C_{\mathrm{e}}=2.1 \times 10^{4} \mathrm{Jm}^{-3} \mathrm{~K}^{-1}, \quad C_{\mathrm{l}}=1.5 \times 10^{6} \mathrm{Jm}^{-3} \mathrm{~K}^{-1}, \\
& S_{\mathrm{e}}=1 \times 10^{12} \mathrm{Wm}^{-3}, \quad S_{\mathrm{l}}=0 \mathrm{Wm}^{-3}, \quad \tau_{F}=10 \mathrm{ps}
\end{aligned}
$$

Figure 2 shows the spatial variation of the difference function $\Delta$ at different times. It is clear that this difference diminishes as $\tau \rightarrow \infty$. This is because hot electrons exchange their energy with the cold lattice and in the limit, as $\tau \rightarrow \infty$, both attain the same temperature.

Figure 3 shows the spatial variation of the electron temperature at different times. The maximum temperature occurs at the surface of the film, which is exposed to the incident laser pulse. As time proceeds, hot electrons near the hot surface give part of their energy to cold electrons, which are far away of the hot surface, and to cold lattice. In the limit, as $\tau \rightarrow \infty$, electron gas and solid lattice attain the same uniform and steady temperature.

It is clear from Fig. 3 that the boundary condition $\left(\partial \theta_{\mathrm{e}} / \partial X\right)(\tau, 0)=0$ is not satisfied at $\tau=5$. This strange behavior is justified due to the fact that the heating source term is assumed to vary in the form $S=S_{0} \delta(\tau) \delta(X)$. This implies that the source evolves all of its energy at $\tau=0$ and $X=0$. At these instant of time and specified location, the electron gas, which absorbs this energy first, has much higher temperature than the solid lattice. This implies that $\theta_{\mathrm{e}} \gg \theta_{1}$, and as a result, the perturbation technique fails near $\tau=0$ and $X=0$. However, for times and locations far from 0 , the difference between $\theta_{\mathrm{e}}$ and $\theta_{\mathrm{l}}$ is small enough to be considered as a perturbed quantity, and as a result, the solution becomes accurate. It is clear from Fig. 3 that the boundary conditions at $\tau \gg 5$ and at $X=1$ and any time are satisfied very well.

In all cases, there is no problem if we have selected source term varies smoothly in time and space. We may assume any form for the heating source term and insert it directly into Eq. (29) and carry out the required integrations.

The reasons for selecting $S=S_{0} \delta(\tau) \delta(X)$, is to simplify the mathematical manipulations and to get closed form solutions. It is worth mentioning here the fact that there are no practical applications involve heating sources vary in the form of $S=S_{0} \delta(\tau) \delta(X)$. It is impossible for a source term to evolve all its energy during a zero time interval and

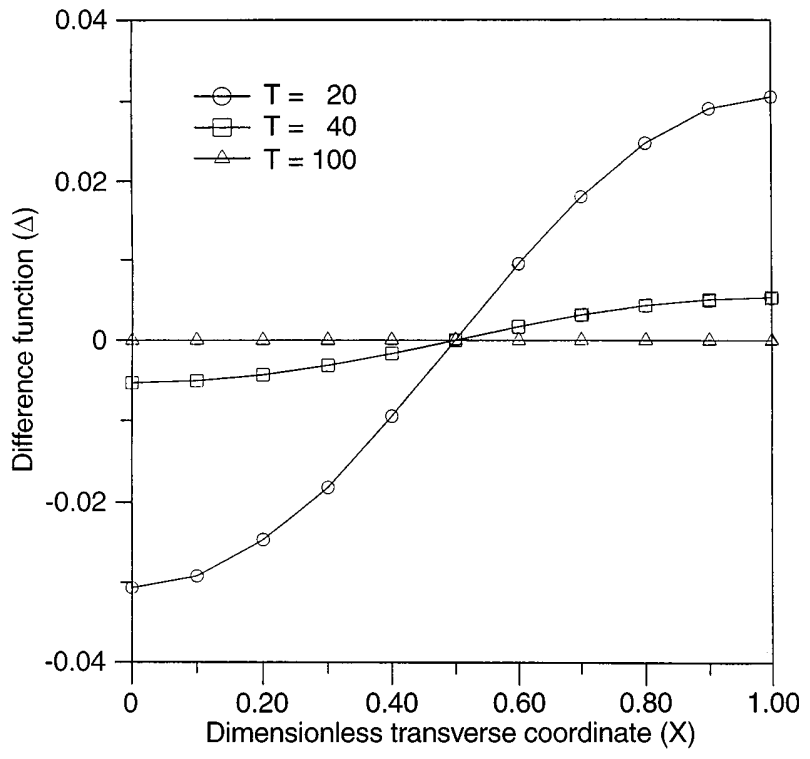

Fig. 2. Transient variation of the difference function

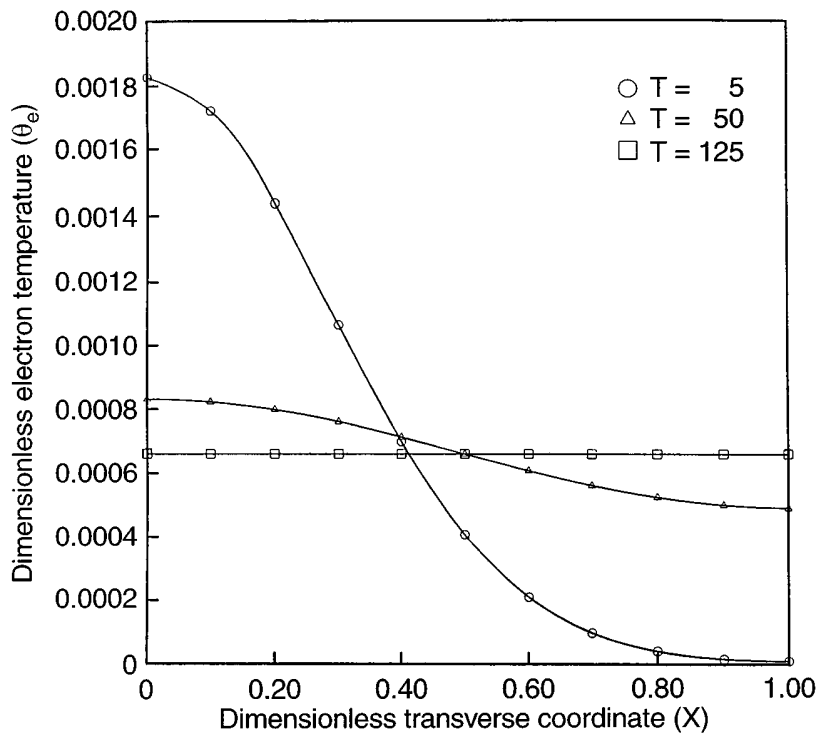

Fig. 3. Transient variation of the dimensionless electron temperature, $\theta_{\mathrm{e}}$

within a zero thickness layer. If the heat source is of non zero time duration and spatial thickness and if the material has large coupling factor, then the difference between $\theta_{\mathrm{e}}$ and $\theta_{\mathrm{l}}$ is small enough to be considered as a perturbed quantity and the strange behavior in Fig. 3 will not appear.

\section{3}

\section{Conclusion}

The hyperbolic two-step model, which describes the thermal behavior of thin metal films under fast rate of heating, is generalized by including diffusion terms in both the electron and lattice energy equations and assuming that incident laser radiation is absorbed by both the electron gas and solid lattice to account for the thermal behaviour of semiconducting and impure metals. A perturbation technique is proposed for solution of the gen- 
eralized equations. The perturbation technique is utilized to eliminate the coupling between the electron and phonon energy equations when the normalized temperature dif-

ference between electrons and phonons is a small quantity, which is true in materials exhibit high coupling factors. The obtained simplified governing equations are solved analytically using Green's function transform to get closed form expressions for the electron and lattice temperatures and electron and lattice heat fluxes.

\section{References}

Al-Nimr MA; Masoud S (1997) Nonequilibrium laser heating of metal films. Heat Transfer 119: 188-190

Al-Nimr MA (1997) Heat transfer mechanisms during short-duration laser heating of thin metal films. Int J Thermophysics 18(5): 1257-1268

Anisimov SL; Kapeliovich BL; Perelman TL (1974) Electron emission from metal surfaces exposed to ultra-short laser pulses. Soviet Physics JETP 39: 375-377

Appleton BR; Cellar GK (eds) (1982) Laser and electron-beam interaction with solids, Elsevier Science, New York

Blakemore JS (1974) Solid State Physics, 2nd Edition, Chp. 3, Cambridge University Press, London

Fujimoto JG; Liu JM; Ippen EP (1984) Femtosecond laser interaction with metallic tungsten and non-equilibrium electron and lattice temperature. Physical Rev Lett 53: $1837-1840$

Ozisik MN (1993) Heat Conduction, 2nd Edition, John Wiley, Chp. 6, pp. 214-256

Qiu TQ; Tien CL (1992) Short-pulse laser heating on metals. Int J Heat and Mass Transfer 35(3): 719-726

Qiu TQ; Tien CL (1993) Heat transfer mechanism during shortpulse laser heating of metals. J Heat Transfer 115: 835-841

Tzou DY; Ozisik MN; Chiffelle RJ (1994) The lattice temperature in the microscopic two-step model. J Heat Transfer 116: $1034-1038$

Tzou DY (1995a) A unified field approach for heat conduction from macro- to micro-scales. J Heat Transfer 117: 8-16

Tzou DY (1995b) The generalized lagging response in small-scale and high-rate heating. Int J Heat Mass Transfer 38(17): 32313240 